\title{
GENERATORS FOR THE MAPPING CLASS GROUP OF A NONORIENTABLE SURFACE
}

\author{
SUSUMU HIROSE
}

\begin{abstract}
We show that Szepietowski's system of generators for the mapping class group of a non-orientable surface is a minimal generating set by Dehn twists and $Y$-homemorphisms.
\end{abstract}

Let $N_{g}$ be a non-orientable surface which is a connected sum of $g$ projective planes. Let $\mathcal{M}\left(N_{g}\right)$ be the group of isotopy classes of homeomorphisms over $N_{g}$, i.e., the mapping class group of $N_{g}$. In this paper, we assume that $g \geq 4$.

We introduce some elements of $\mathcal{M}\left(N_{g}\right)$. A simple closed curve $\gamma_{1}\left(\right.$ resp. $\left.\gamma_{2}\right)$ in $N_{g}$ is two-sided (resp. one-sided) if a regular neighborhood of $\gamma_{1}$ (resp. $\gamma_{2}$ ) is an annulus (resp. Möbius band). For a two-sided simple closed curve $\gamma$ on $N_{g}$, we denote by $t_{\gamma}$ a Dehn twist about $\gamma$. We indicate the direction of a Dehn twist by an arrow beside the curve $\gamma$ as shown in Figure 1. For a one-sided simple closed curve $m$ and a two-sided simple closed curve $a$ which intersect transversely in one point, let $K \subset N_{g}$ be a regular neighborhood of $m \cup a$, which is homeomorphic to the Klein bottle with one boundary component. Let $M$ be a regular neighborhood of $m$. We denote by $Y_{m, a}$ a homeomorphism over $N_{g}$ which is described as the result of pushing $M$ once along $a$ keeping the boundary of $K$ fixed (see Figure 2). We call $Y_{m, a}$ a $Y$-homeomorphism.

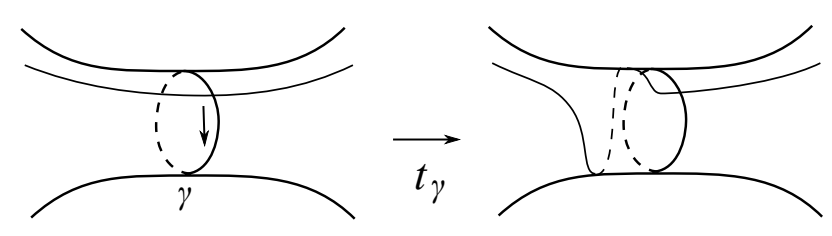

FiguRE 1 . The direction of $t_{\gamma}$ is indicated by an arrow beside $\gamma$.

Lickorish showed that $\mathcal{M}\left(N_{g}\right)$ is generated by Dehn twists and $Y$-homeomorphisms [7], and that $\mathcal{M}\left(N_{g}\right)$ is not generated by Dehn twists [8]. Furthermore, Chillingworth

This research was supported by Grant-in-Aid for Scientific Research (C) (No. 16K05156), Japan Society for the Promotion of Science. 


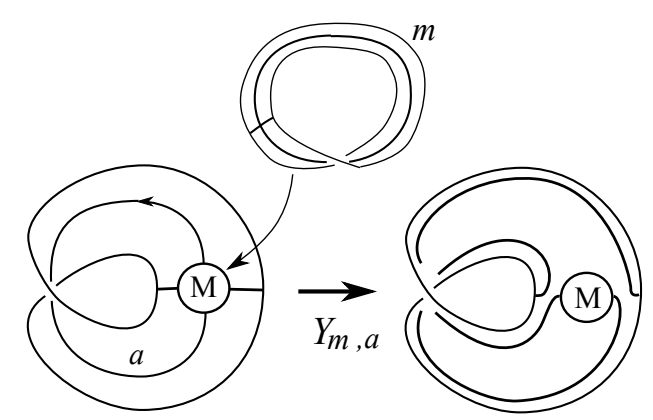

Figure 2. A cirlcle with "M" indicates a place where to attach a Möbius band.

[2] found a finite system of generators for $\mathcal{M}\left(N_{g}\right)$. Birman and Chillingworth [1] obtained a finite system of generators by using an argument on the orientable two fold covering of $N_{g}$. Szepietowski 9] reduced the system of Chillingworth's generators for $\mathcal{M}\left(N_{g}\right)$ and showed:

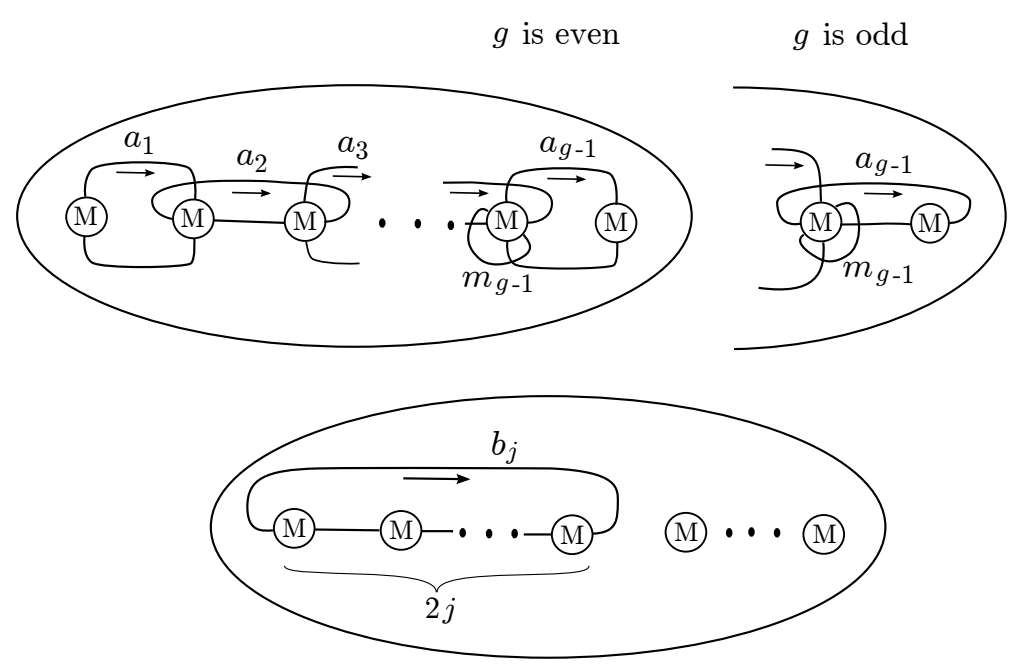

Figure 3. Chillingworth's generators for $\mathcal{M}\left(N_{g}\right)$.

Theorem 1. [9, Theorem 3.1] $\mathcal{M}\left(N_{g}\right)$ is generated by $t_{a_{i}}(i=1, \ldots, g-1), t_{b_{2}}$ and $Y_{m_{g-1}, a_{g-1}}$, where $a_{i}, m_{g-1}, b_{2}$ are simple closed curves shown in Figure 3 .

On the other hand, Lickorish [6] showed that the mapping class group $\mathcal{M}\left(\Sigma_{g}\right)$ of the orientable closed surface $\Sigma_{g}$ of genus $g$ is generated by finitely many Dehn twists, and Humphries [4] reduced the number of Dehn twists generating $\mathcal{M}\left(\Sigma_{g}\right)$ to $2 g+1$ and 
showed that this is the minimum number of Dehn twists generating $\mathcal{M}\left(\Sigma_{g}\right)$. We will show the analogous result for the mapping class group of the non-orientable surface.

Theorem 2. We assume $g \geq 4$. If Dehn twists $t_{c_{1}}, \ldots, t_{c_{n}}$ and $Y$-homeomorphisms $Y_{1}, \ldots, Y_{k}$ generate $\mathcal{M}\left(N_{g}\right)$, then $n \geq g$ and $k \geq 1$. In particular, any proper subset of $\left\{t_{a_{i}}(i=1, \ldots, g-1), t_{b_{2}}, Y_{m_{g-1}, a_{g-1}}\right\}$ does not generate $\mathcal{M}\left(N_{g}\right)$.

Remark 3. When $g=1, \mathcal{M}\left(N_{g}\right)$ is trivial. When $g=2, \mathcal{M}\left(N_{2}\right) \cong \mathbb{Z}_{2} \times \mathbb{Z}_{2}$ and generated by $t_{a_{1}}$ and $Y_{m_{1}, a_{1}}$ (see [7, Lemma 5]), therefore $\left\{t_{a_{1}}, Y_{m_{1}, a_{1}}\right\}$ is a minimal generating set by Dehn twists and $Y$-homeomorphisms. When $g=3, \mathcal{M}\left(N_{3}\right)$ is generated by $t_{a_{1}}, t_{a_{2}}$ and $Y_{m_{2}, a_{2}}$ (see [1, Theorem 3] and [9, Theorem 3.1]). If $\mathcal{M}\left(N_{3}\right)$ is generated by one Dehn twist $t_{a}$ and one $Y$-homeomorphism, then the group of the action of $\mathcal{M}\left(N_{3}\right)$ on $H_{1}\left(N_{3} ; \mathbb{Z}_{2}\right)$ should be isomorphic to $\mathbb{Z}_{2}$ generated by the induced isomorphism $\left(t_{a}\right)_{*}$ on $H_{1}\left(N_{3} ; \mathbb{Z}_{2}\right)$. Nevertheless, $\left(t_{a_{1}}\right)_{*}$ is not equal to $\left(t_{a_{2}}\right)_{*}$. Therefore $\left\{t_{a_{1}}, t_{a_{2}}, Y_{m_{2}, a_{2}}\right\}$ is a minimal generating set by Dehn twists and $Y$-homeomorphisms.

Let $w_{1}: H_{1}\left(N_{g} ; \mathbb{Z}_{2}\right) \rightarrow \mathbb{Z}_{2}$ be the first Stiefel-Whitney class, that is to say, if $x \in H_{1}\left(N_{g} ; \mathbb{Z}_{2}\right)$ is represented by a one-sided simple closed curve on $N_{g}$ then $w_{1}(x)=1$, otherwise $w_{1}(x)=0$. For the basis $\left\{x_{1}, \ldots, x_{g}\right\}$ for $H_{1}\left(N_{g} ; \mathbb{Z}_{2}\right)$ indicated in Figure 4 , $w_{1}\left(x_{i}\right)=1$. For each pair of elements $x, y$ of $H_{1}\left(N_{g} ; \mathbb{Z}_{2}\right)$, the $\mathbb{Z}_{2}$-intersection form of $x$ and $y$ is denoted by $(x, y)$. For the basis $\left\{x_{1}, \ldots, x_{g}\right\},\left(x_{i}, x_{j}\right)=\delta_{i, j}$. Let $H_{1}^{+}\left(N_{g} ; \mathbb{Z}_{2}\right)$ be the kernel of $w_{1}$, then $\operatorname{dim}_{\mathbb{Z}_{2}} H_{1}^{+}\left(N_{g} ; \mathbb{Z}_{2}\right)=g-1$. If a complement of a two-sided simple closed curve $c$ on $N_{g}$ is connected and non-orientable, we call $c$ an admissible $A$-circle. For an admissible $A$-circle $c$ on $N_{g}, N_{g} \backslash c$ is homeomorphic to $N_{g-2}$ removed two 2-disks. Therefore, if $c_{1}$ and $c_{2}$ are admissible $A$-circles then there is $\phi \in \mathcal{M}\left(N_{g}\right)$ such that $\phi\left(c_{1}\right)=c_{2}$, by the change of coordinates principle in [3, $\left.\S 1.3\right]$.

Lemma 4. Let c be a two-sided simple closed curves on $N_{g}$. If c is not admissible, then $c$ represent 0 or $x_{1}+\cdots+x_{g}$ in $H_{1}\left(N_{g} ; \mathbb{Z}_{2}\right)$.

Proof. If $c$ is not admissible, then either $N_{g} \backslash c$ is not connected or $N_{g} \backslash c$ is connected and orientable. In the former case, $c$ is 0 in $H_{1}\left(N_{g} ; \mathbb{Z}_{2}\right)$. In the latter case, $g$ is even, and there is a homeomorphism which brings $c$ to $b_{g / 2}$ in Figure 3 , since their complements are homeomorphic to $\Sigma_{g / 2-1}$ removed two 2-disks. The simple closed 


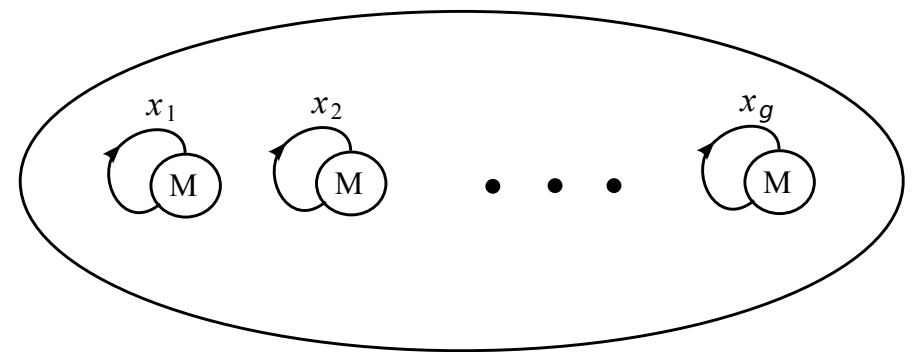

FiguRE 4 . A basis for $H_{1}\left(N_{g} ; \mathbb{Z}_{2}\right)$.

curve $b_{g / 2}$ represents $x_{1}+\cdots+x_{g}$ and the action of any homeomorphism of $N_{g}$ on $H_{1}\left(N_{g} ; \mathbb{Z}_{2}\right)$ preserves $x_{1}+\cdots+x_{g}$. Therefore $c$ represents $x_{1}+\cdots+x_{g}$.

Lemma 5. If $t_{c_{1}}, \ldots, t_{c_{n}}$ and $Y$-homeomorphisms $Y_{1}, \ldots, Y_{k}$ generate $\mathcal{M}\left(N_{g}\right)$, then at least one of $c_{1}, \ldots, c_{n}$ is admissible.

Proof. For $y \in H_{1}\left(N_{g} ; \mathbb{Z}_{2}\right)$, we define an isomorphism $\tau_{y}$ of $H_{1}\left(N_{g} ; \mathbb{Z}_{2}\right)$ by $\tau_{y}(x)=$ $x+(x, y) y$. By Lemma 4 and the fact that $Y$-homeomorphisms acts on $H_{1}\left(N_{g} ; \mathbb{Z}_{2}\right)$ trivially, if $c_{1}, \ldots, c_{n}$ are not admissible, then the action of each elements of $\mathcal{M}\left(N_{g}\right)$ on $H_{1}\left(N_{g} ; \mathbb{Z}_{2}\right)$ is a power of $\tau_{x_{1}+\cdots+x_{g}}$. On the other hand, $\left(t_{a_{1}}\right)_{*}=\tau_{x_{1}+x_{2}}$ is not a power of $\tau_{x_{1}+\cdots+x_{g}}$.

Lemma 6. If $t_{c_{1}}, \ldots, t_{c_{n}}$ and $Y$-homeomorphisms $Y_{1}, \ldots, Y_{k}$ generate $\mathcal{M}\left(N_{g}\right)$ then $\left[c_{1}\right], \ldots,\left[c_{n}\right]$ generate $H_{1}^{+}\left(N_{g} ; \mathbb{Z}_{2}\right)$. In particular, $n \geq g-1$.

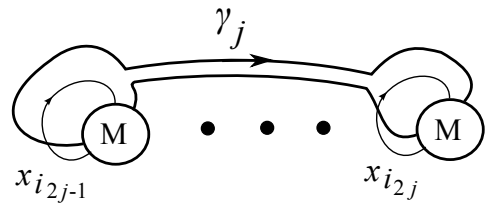

FiguRE 5. An element $x_{i_{2 j-1}}+x_{i_{2 j}} \in H_{1}\left(N_{g} ; \mathbb{Z}_{2}\right)$ is represented by an admissible $A$-circle $\gamma_{i}$

Proof. By Lemma 5, we may assume $c_{1}$ is an admissible $A$-circle. For any $x \in$ $H_{1}^{+}\left(N_{g} ; \mathbb{Z}_{2}\right)$, we can write $x=x_{i_{1}}+x_{i_{2}}+\cdots+x_{i_{2 k}}$. We can represent $x_{i_{2 j-1}}+x_{i_{2 j}}$ by an admissible $A$-circle $\gamma_{j}$ as in Figure 5. Hence, $x$ is represented by a union of admissible $A$-circles, that is, $x=\left[\gamma_{1}\right]+\cdots+\left[\gamma_{k}\right]$ in $H_{1}^{+}\left(N_{g} ; \mathbb{Z}_{2}\right)$. For each $\gamma_{j}$, there is an element $\phi_{j} \in \mathcal{M}\left(N_{g}\right)$ such that $\phi_{j}\left(c_{1}\right)=\gamma_{j}$. By the assumption of this lemma, $\phi_{j}$ 
is a product of $t_{c_{1}}, \ldots, t_{c_{n}}$ and $Y_{1}, \ldots, Y_{k}$. We see that $Y_{i}$ acts on $H_{1}\left(N_{g} ; \mathbb{Z}_{2}\right)$ trivially, and, for each $x \in H_{1}\left(N_{g} ; \mathbb{Z}_{2}\right),\left(t_{c_{i}}\right)_{*}(x)=x+\left(x,\left[c_{i}\right]\right)\left[c_{i}\right]$. Therefore, $\left[\gamma_{j}\right] \in H_{1}^{+}\left(N_{g} ; \mathbb{Z}_{2}\right)$ is a sum of $\left[c_{1}\right], \ldots,\left[c_{n}\right]$, hence $x$ is a sum of $\left[c_{1}\right], \ldots,\left[c_{n}\right]$. This shows that $H_{1}^{+}\left(N_{g} ; \mathbb{Z}_{2}\right)$ is generated by $\left[c_{1}\right], \ldots,\left[c_{n}\right]$.

Let $2 \times: \mathbb{Z}_{2} \rightarrow \mathbb{Z}_{4}$ be an injection defined by $2 \times([n])=[2 n]$. A map $q$ : $H_{1}\left(N_{g} ; \mathbb{Z}_{2}\right) \rightarrow \mathbb{Z}_{4}$ is called a $\mathbb{Z}_{4}$-quadratic form, if $q(x+y)=q(x)+q(y)+2 \times(x, y)$ for any $x, y \in H_{1}\left(N_{g} ; \mathbb{Z}_{2}\right)$. This map $q$ is determined by values of $q$ for elements in a $\mathbb{Z}_{2}$-basis of $H_{1}\left(N_{g} ; \mathbb{Z}_{2}\right)$. Putting $x=y=0$ in the above formula, we have $q(0)=0$. If $x \in H_{1}\left(N_{g} ; \mathbb{Z}_{2}\right)$ is represented by a one-sided simple closed curve, in other word $x$ is represented by a core of a Möbius band embedded in $N_{g}$, then $(x, x)=1$. Since $2 x=0$ in $H_{1}\left(N_{g} ; \mathbb{Z}_{2}\right)$, we have $0=q(x+x)=q(x)+q(x)+2 \times(x, x)=2 q(x)+2$. Therefore, we have $q(x)= \pm 1$. By the same argument, if $x \in H_{1}\left(N_{g} ; \mathbb{Z}_{2}\right)$ is represented by a two-sided simple closed curve, then we have $q(x)=0$ or 2 .

Lemma 7. There is no $\mathbb{Z}_{4}$-quadratic form over $H_{1}\left(N_{g} ; \mathbb{Z}_{2}\right)$ which is preserved by every non-trivial element of $\mathcal{M}\left(N_{g}\right)$.

Proof. For any $\mathbb{Z}_{4}$-quadratic form $q$ over $H_{1}\left(N_{g} ; \mathbb{Z}_{2}\right)$, there is a non-trivial element $x \in H_{1}^{+}\left(N_{g} ; \mathbb{Z}_{2}\right)$ such that $q(x)=0$; even if $q\left(\left[a_{1}\right]\right)=q\left(\left[a_{3}\right]\right)=2$ then $q\left(\left[a_{1}\right]+\left[a_{3}\right]\right)=$ $q\left(\left[a_{1}\right]\right)+q\left(\left[a_{3}\right]\right)+2 \times\left(\left[a_{1}\right],\left[a_{3}\right]\right)=0$. When $x=x_{i_{1}}+\ldots+x_{i_{2 n}}$, let $y=x_{i_{1}}$, then $(y, x)=1$. Let $\gamma$ be a simple closed curve on $N_{g}$ representing $x$ then $q \circ\left(t_{\gamma}\right)_{*}(y)=$ $q(y+(y, x) x)=q(y)+q((y, x) x)+2 \times(y,(y, x) x)=q(y)+q(x)+2=q(y)+2 \neq q(y)$. Therefore $q \circ\left(t_{\gamma}\right)_{*} \neq q$.

Lemma 8. Let $c_{1}, \ldots, c_{g-1}$ be two-sided simple closed curves such that $\left[c_{1}\right], \ldots,\left[c_{g-1}\right]$ generate $H_{1}^{+}\left(N_{g} ; \mathbb{Z}_{2}\right)$, then there is a $\mathbb{Z}_{4}$-quadratic form over $H_{1}\left(N_{g} ; \mathbb{Z}_{2}\right)$ preserved by any $t_{c_{i}}$.

Proof. Let $\alpha$ be a one-sided simple closed curve on $N_{g}$, then $\left\{\left[c_{1}\right], \ldots,\left[c_{g-1}\right],[\alpha]\right\}$ is a $\mathbb{Z}_{2}$-basis of $H_{1}\left(N_{g} ; \mathbb{Z}_{2}\right)$. We define a $\mathbb{Z}_{4}$-quadratic form $q$ over $H_{1}\left(N_{g} ; \mathbb{Z}_{2}\right)$ by $q\left(\left[c_{1}\right]\right)=$ $\cdots=q\left(\left[c_{g-1}\right]\right)=2$ and $q([\alpha])=1$. For any $i=1, \ldots, g-1$ and $x \in H_{1}\left(N_{g} ; \mathbb{Z}_{2}\right)$, we see $q \circ\left(t_{c_{i}}\right)_{*}(x)=q\left(x+\left(x,\left[c_{i}\right]\right)\left[c_{i}\right]\right)=q(x)+q\left(\left(x,\left[c_{i}\right]\right)\left[c_{i}\right]\right)+2 \times\left(x,\left(x,\left[c_{i}\right]\right)\left[c_{i}\right]\right)$. If $\left(x,\left[c_{i}\right]\right)=0, q \circ\left(t_{c_{i}}\right)_{*}(x)=q(x)$. If $\left(x,\left[c_{i}\right]\right)=1, q \circ\left(t_{c_{i}}\right)_{*}(x)=q(x)+q\left(\left[c_{i}\right]\right)+2 \times$ $\left(x,\left[c_{i}\right]\right)=q(x)+2+2=q(x)$. Therefore, $q \circ\left(t_{c_{i}}\right)_{*}=q$ for any $i=1, \ldots, g-1$. 
We assume that Dehn twists $t_{c_{1}}, \ldots, t_{c_{n}}$ and $Y$-homeomorphisms $Y_{1}, \ldots, Y_{k}$ generate $\mathcal{M}\left(N_{g}\right)$. In [8], Lickorish showed that $\mathcal{M}\left(N_{g}\right)$ is not generated by Dehn twists, therefore we see $k \geq 1$. By Lemma 6 , $\left[c_{1}\right], \ldots,\left[c_{n}\right]$ generate $H_{1}^{+}\left(N_{g} ; \mathbb{Z}_{2}\right)$, in particular $n \geq g-1$. We assume that $n=g-1$. By Lemma 8 , there is a $\mathbb{Z}_{4}$-quadratic form over $H_{1}\left(N_{g} ; \mathbb{Z}_{2}\right)$ preserved by Dehn twists $t_{c_{1}}, \ldots, t_{c_{n}}$ and $Y$-homeomorphisms $Y_{1}, \ldots, Y_{k}$, which contradicts Lemma 17. Hence, we see $n \geq g$. This completes the proof of Theorem 2 .

Remark 9. The proof of Theorem 2 is inspired by the master thesis [5] by Shigehisa Ishimura, in which he proved Humphries' result by using $\mathbb{Z}_{2}$-quadratic form over $H_{1}\left(\Sigma_{g} ; \mathbb{Z}_{2}\right)$.

Acknowledgments. The author would like to thank Mustafa Korkmaz and Genki Omori for their useful comments.

\section{REFERENCES}

[1] J.S. Birman and D. R. J. Chillingworth, On the homeotopy group of a non-orientable surface, Math. Proc. Camb. Phil. Soc. 71 (1972), 437-448. Erratum: Math. Proc. Camb. Phil. Soc. 136 (2004), 441-441.

[2] D. R. J. Chillingworth, A finite set of generators for the homeotopy group of a non-orientable surface, Proc. Camb. Phil. Soc. 65 (1969), 409-430

[3] B. Farb and D. Margalit, A primer on mapping class groups. Princeton Mathematical Series, 49. Princeton University Press, Princeton, NJ, 2012.

[4] S. P. Humphries, Generators for the mapping class group, In: Topology of low-dimensional manifolds (Proc. Second Sussex Conf., Chelwood Gate, 1977), 44-47, Lecture Notes in Math., 722, Springer, Berlin, 1979.

[5] S. Ishimura, The spin structures over surfaces and the number of generators for the mapping class group, (Japanese) Master Thesis, Osaka City University, 2004.

[6] W.B.R. Lickorish, A finite set of generators for the homeotopy group of a 2-manifold, Proc. Cambridge Philos. Soc. 60(1964), 769-778, Erratum: Proc. Cambridge Philos. Soc. 62(1966), 679-681.

[7] W.B.R. Lickorish, Homeomorphisms of non-orientable two-manifolds, Proc. Cambridge Philos. Soc. 59(1963), 307-317.

[8] W.B.R. Lickorish, On the homeomorphisms of a non-orientable surface, Proc. Cambridge Philos. Soc. 61(1965), 61-64.

[9] B. Szepietowski, A finite generating set for the level 2 mapping class group of a nonorientable surface, Kodai Math. J. 36(2013), 1-14

Department of Mathematics, Faculty of Science and Technology, Tokyo UniverSity of Science, NodA, Chiba, 278-8510, JaPAN

E-mail address: hirose_susumu@ma.noda.tus.ac.jp 